\title{
On Kleiman-Piene's question for Gauss maps
}

\author{
Satoru Fukasawa
}

\begin{abstract}
We study the product of a Fermat hypersurface $X_{0}^{p+1}+\cdots+X_{n}^{p+1}=0 \subset \mathbf{P}^{n}$ with $n \geqslant 3$ and $\mathbf{P}^{1}$, embedded in $\mathbf{P}^{2 n+1}$ by Segre embedding where $p>0$ is the characteristic of the base field. This smooth variety is nonreflexive and has Gauss map which is an embedding. This gives a negative answer to the following Kleiman-Piene question in any positive characteristic: does the separability of the Gauss map imply reflexivity? The only known smooth examples, which give a negative answer, are given by Kaji in characteristic 2 .
\end{abstract}

\section{Introduction}

Let $X \subset \mathbf{P}^{N}$ be a smooth projective variety of dimension $n, C X$ be the conormal variety $\{(x, H) \in$ $\left.X \times \mathbf{P}^{N^{*}} \mid \mathbf{T}_{x} X \subset H\right\} \subset X \times \mathbf{P}^{N^{*}}$, where $\mathbf{T}_{x} X$ is the projective embedded tangent space at a point $x$, with the natural projection $p_{2}: C X \rightarrow \mathbf{P}^{N^{*}}$, and let $X^{*}=p_{2}(C X)$ be its dual. The Gauss map $\gamma$ on $X$ is the morphism from $X$ to the Grassmannian $\mathbf{G}(n, N) \cong \mathbf{G}^{*}(N-n-1, N)$ which assigns to a point $x \in X$ the projective tangent space $\mathbf{T}_{x} X \in \mathbf{G}(n, N)$, or its dual $\left(\mathbf{T}_{x} X\right)^{*} \in \mathbf{G}^{*}(N-n-1, N)$. Now we study $\gamma: X \rightarrow \mathbf{G}^{*}(N-n-1, N)$. We have the diagram

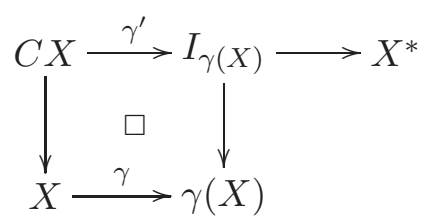

where $I_{\gamma(X)}=\left\{(E, H) \in \gamma(X) \times \mathbf{P}^{N^{*}} \mid H \in E\right\} \subset \gamma(X) \times \mathbf{P}^{N^{*}}$ and $\gamma^{\prime}(x, H)=\left(\left(\mathbf{T}_{x} X\right)^{*}, H\right)$. Then, Kleiman and Piene raised the following question.

Question [KP91, pp. 108-109]. Is $I_{\gamma(X)} \rightarrow X^{*}$ separable?

If $\operatorname{dim} \gamma(X)=1$, then it is known that $I_{\gamma(X)} \rightarrow X^{*}$ is always separable [Fuk05, Kaj92].

Nonreflexive projective varieties with separable Gauss maps give a negative answer. ( $X$ is called reflexive if $C X \rightarrow X^{*}$ is separable [Kle86].) In characteristic 2, the first such varieties were found by Kaji [Kaj03]. He studied Segre varieties (i.e. products of projective spaces embedded by Segre embeddings) and their duals. He proved that some odd-dimensional Segre varieties, for example $\mathbf{P}^{1} \times \mathbf{P}^{1} \times \mathbf{P}^{1}$, are not reflexive in characteristic 2 and have Gauss maps that are embeddings. If we do not need the smoothness of $X$, then the present author already found nonreflexive varieties with birational Gauss maps in any positive characteristic [Fuk].

In this paper we study the product of a Fermat hypersurface $X_{0}^{p+1}+\cdots+X_{n}^{p+1}=0 \subset \mathbf{P}^{n}$ and $\mathbf{P}^{1}$, embedded in $\mathbf{P}^{2 n+1}$ by Segre embedding where $p>0$ is the characteristic of the base field. This smooth variety, call it $X$, has a Gauss map which is an embedding and inseparable morphism $I_{\gamma(X)} \rightarrow X^{*}$ when $n \geqslant 3$. Consequently, $X$ is nonreflexive if $n \geqslant 3$. The author thinks that this

Received 13 January 2006, accepted in final form 4 April 2006.

2000 Mathematics Subject Classification 14N05.

Keywords: conormal variety, dual variety, Fermat hypersurface, Gauss map, reflexivity.

This journal is (C) Foundation Compositio Mathematica 2006. 


\section{S. FUKASAWA}

is the first smooth example, in characteristic $p>2$ or of even dimension, which gives a negative answer to the Kleiman-Piene's question.

We work over an algebraically closed field $K$ of characteristic $p>0$. Varieties are integral algebraic schemes over $K$. Here $[v] \in \mathbf{P}^{N}$ denotes the point of $\mathbf{P}^{N}$ corresponding to the equivalence class of $v \in \mathbf{A}^{N+1} \backslash 0$.

\section{Product of a Fermat hypersurface and the projective line}

Let $Y \subset \mathbf{P}^{n}$ with $n \geqslant 3$ be a Fermat hypersurface given by $X_{0}^{p+1}+\cdots+X_{n}^{p+1}=0$, and $X=Y \times \mathbf{P}^{1} \subset$ $\mathbf{P}^{2 n+1}$ embedded by Segre embedding. Let $\left(1: x_{1}: \cdots: x_{n}\right)$ be an affine coordinates of $Y$ and $(1: u)$ be of $\mathbf{P}^{1}$, then $X$ is (the closure of) the image of $f: Y \times \mathbf{P}^{1} \rightarrow \mathbf{P}^{2 n+1} ;\left(1: x_{1}: \cdots: x_{n}\right) \times(1: u) \mapsto$ $\left(1: x_{1}: \cdots: x_{n-1}: u: x_{n}: x_{1} u: \cdots: x_{n} u\right)$. We take $x_{1}, \ldots, x_{n-1}$ as a system of local coordinates of $Y$ (i.e. the function field $K(Y)$ is separable algebraic over $K\left(x_{1}, \ldots, x_{n-1}\right)$ ). The projective tangent space at $f\left(x_{1}, \ldots, x_{n}, u\right)$ is spanned by the $n+1$ row vectors of the following matrices:

$$
\left(\begin{array}{cccccccccc}
1 & x_{1} & \ldots & x_{n-1} & u & x_{n} & x_{1} u & \ldots & x_{n-1} u & x_{n} u \\
0 & 1 & \ldots & 0 & 0 & \frac{\partial x_{n}}{\partial x_{1}} & u & \ldots & 0 & \frac{\partial x_{n}}{\partial x_{1}} u \\
\vdots & \vdots & \ddots & \vdots & \vdots & \vdots & \vdots & \ddots & \vdots & \vdots \\
0 & 0 & \ldots & 1 & 0 & \frac{\partial x_{n}}{\partial x_{n-1}} & 0 & \ldots & u & \frac{\partial x_{n}}{\partial x_{n-1}} u \\
0 & 0 & \ldots & 0 & 1 & 0 & x_{1} & \ldots & x_{n-1} & x_{n}
\end{array}\right) \sim\left(I_{n+1}\right.
$$

where $I_{n+1}$ is the $(n+1) \times(n+1)$ unit matrix and

$$
A=\left(\begin{array}{ccccc}
x_{n}-\sum_{j=1}^{n-1} \frac{\partial x_{n}}{\partial x_{j}} x_{j} & -x_{1} u & \ldots & -x_{n-1} u & -\sum_{j=1}^{n-1} \frac{\partial x_{n}}{\partial x_{j}} x_{j} u \\
\frac{\partial x_{n}}{\partial x_{1}} & u & \ldots & 0 & \frac{\partial x_{n}}{\partial x_{1}} u \\
\vdots & \vdots & \ddots & \vdots & \vdots \\
\frac{\partial x_{n}}{\partial x_{n-1}} & 0 & \ldots & u & \frac{\partial x_{n}}{\partial x_{n-1}} u \\
0 & x_{1} & \ldots & x_{n-1} & x_{n}
\end{array}\right) .
$$

We also have

$$
\frac{\partial x_{n}}{\partial x_{j}}=-\frac{x_{j}^{p}}{x_{n}^{p}}
$$

for $j=1, \ldots, n-1$. These imply that the Gauss map on $X$ is an embedding. Calculation of the dual vector space shows that $\gamma(X) \subset \mathbf{G}^{*}(n, 2 n+1)$ is locally represented by the matrix $B=\left(\begin{array}{ll}-{ }^{\mathrm{t}} A & I_{n+1}\end{array}\right)$. Let $\rho_{i}$ be the $(i+1)$ th row vector of $B$ for $0 \leqslant i \leqslant n$. By using a local trivialization, $I_{\gamma(X)} \rightarrow X^{*}$ is generically identified with the morphism $g: Y_{0} \times \mathbf{A}^{1} \times \mathbf{P}^{n} \rightarrow X^{*}$; $\left(x_{1}, \ldots, x_{n}\right) \times(u) \times\left(t_{0}: \cdots: t_{n}\right) \mapsto\left[t_{0} \rho_{0}+\cdots+t_{n} \rho_{n}\right]$ where $Y_{0}$ is an affine locus of $Y$ with $X_{0} X_{n} \neq 0$. The affine lifting is $\hat{g}: Y_{0} \times \mathbf{A}^{1} \times \mathbf{A}^{n+1} \rightarrow \widehat{X^{*}} ;\left(x_{1}, \ldots, x_{n}\right) \times(u) \times\left(t_{0}, \ldots, t_{n}\right) \mapsto t_{0} \rho_{0}+\cdots+t_{n} \rho_{n}$ where $\widehat{X^{*}}$ is the affine cone of $X^{*}$. By easy computation, we have

$$
\frac{\partial \hat{g}}{\partial x_{j}}=\left(t_{j}+t_{n} \frac{\partial x_{n}}{\partial x_{j}}\right) \mathbf{u}
$$

where $\mathbf{u}={ }^{\mathrm{t}}(u, 0, \ldots, 0,-1,0, \ldots, 0)$. This implies that the rank of the differential of $g$ is $n+2$, and hence drops when $n \geqslant 3$. We can easily check that $X^{*}$ is a hypersurface, hence $I_{\gamma(X)} \rightarrow X^{*}$ is inseparable when $n \geqslant 3$. 


\section{KLEIMAN-PIENE'S QUESTION}

\section{ACKNOWLEDGEMENTS}

The author is most grateful to Professor Shun-ichi Kimura and Professor Hajime Kaji for thoughtful and helpful advice.

\section{REFERENCES}

Fuk05 S. Fukasawa, Developable varieties in positive characteristic, Hiroshima Math. J. 35 (2005), 167-182.

Fuk S. Fukasawa, A remark on Kleiman-Piene's question for Gauss maps, Comm. Algebra, to appear.

Kaj92 H. Kaji, On the inseparable degrees of the Gauss map and the projection of the conormal variety to the dual of higher order for space curves, Math. Ann. 292 (1992), 529-532.

Kaj03 H. Kaji, On the duals of Segre varieties, Geom. Dedicata 99 (2003), 221-229.

Kle86 S. L. Kleiman, Tangency and duality, in Proceedings of the 1984 Vancouver Conference in Algebraic Geometry, CMS Conference Proceedings, vol. 6 (American Mathematical Society, Providence, RI, 1986), 163-226.

KP91 S. Kleiman and R. Piene, On the inseparability of the Gauss map, Contemporary Mathematics, vol. 123 (American Mathematical Society, Providence, RI, 1991), 107-129.

Satoru Fukasawa sfuka@hiroshima-u.ac.jp

Department of Mathematics, Hiroshima University, Kagamiyama 1-3-1, Higashi-Hiroshima 7398526, Japan 